\title{
Multi-partite entanglement in Davies environment
}

\author{
Konrad Jałowiecki ${ }^{1}$ and Jerzy Dajka ${ }^{1,2, a}$ \\ ${ }^{1}$ Institute of Physics, University of Silesia in Katowice, Katowice, Poland \\ ${ }^{2}$ Silesian Center for Education and Interdisciplinary Research, University of Silesia \\ in Katowice, Chorzów, Poland
}

Received 7 April 2018

Published online 21 February 2019

\begin{abstract}
We analyse dynamics of genuinely multi-partite entanglement of $\mathrm{N}$-qubit states initially prepared in the form of so called $\mathrm{X}$-matrices with one qubit coupled to a Davies-type environment. We develop an analytic formula for genuinely multi-partite concurrence of the investigated states as a function of time and analyze its time evolution with an emphasis on the qualitative difference between systems affected by a pure decoherence only and those which do dissipate energy at finite temperature.
\end{abstract}

\section{Introduction}

An effect of the omnipresent decoherence [1] has to be taken into account by all who consider quantum mechanics as a modern and useful resource for information processing. The celebrated entanglement [2] is (probably) the most "quantum" resource with a wide spectrum of applications starting from quantum communication (e.g. teleportation), cryptography (key management via the Ekert-like protocols) and quantum computation based on multi-partite entangled cluster states [3]. Since all the protocols act on the stage of real world at finite temperature, coupled to environment, it is of vital importance to verify their effectiveness under an influence of noise.

Various aspects of entanglement of open quantum systems is an object of intensive studies [4] both in a context of bi- and multi-partite entanglement. The latter is 'harder' to study simply because it is harder to define [5]. There are various classes of entangled multi-partite systems: all of them useful but often very different. Except the fully classified 3-partite case [6] one deals with a hard problems of identifying various types of states with non-classical correlations.

In this work, we focus on a certain type of multi-partite entanglement - the genuine multi-partite entanglement [7] which can be effectively characterized by its negativity [8] or concurrence [9]. We study an effect of decoherence modelled by the Davies approximation [10] which, except mathematical rigour, has a well established physical setting [11]. The Davies method has been successfully used in recent studies of various problems in quantum information and physics of open quantum systems including teleportation [12], bi-partite entanglement dynamics [13], quantum discord [14,15], quantum cloning [16], Bell-type [14] and Leggett-Garg non-locality [17], properties

\footnotetext{
a e-mail: jerzy.dajka@us.edu.pl
} 
of geometric phases of qubits [18], thermodynamic properties of nano-systems [19] and quantum games [20]. Our present work continues and supplements our previous studies of quantum phenomena in a presence of Davies environment modelled via the Davies maps [21] related to quantum information processing.

\section{Davies decoherece and entanglement}

Quantum decoherence of, in particular, qubits is caused by the environment. Its influence on the qubit $Q$ is modeled by the Hamiltonian in the form $H=H_{Q}+H_{\text {env }}+H_{\text {int }}$ where $H_{Q}$ is the Hamiltonian of the qubit, $H_{Q}=\frac{\omega}{2}(|1\rangle\langle 1|-| 0\rangle\langle 0|)$, with $\omega$ the energy splitting of the qubit and $|0\rangle,|1\rangle$ spanning a Hilbert space of $Q, H_{\text {env }}$ models the environment and $H_{\text {int }}$ describes the qubit-environment interaction. In this work we assume that the interaction between the qubit and its environment satisfies the Davies weak coupling conditions [10,22] allowing for rigorous construction of the qubit reduced (with respect to the environment) dynamics. It is a completely positive (strictly Markovian) semigroup built in terms of parameters of the microscopic Hamiltonian of the full system [22]. The Davies semigroups can be rigorously and consistently derived from microscopic models of open systems and they satisfy thermodynamic and statistical-mechanical properties of open quantum systems such as the detailed balance condition and the Gibbs canonical distribution in the stationary regime $[11,22]$.

In this paper, we consider only certain elements of Davies semi-groups: the so called Davies maps $[12,14,15,17,21]$ which are completely positive and describe time evolution of qubits (two-level systems with an energy splitting $\omega$ ) coupled to a thermal environment $[10,11]$ via a mapping [21] acting on single qubit density matrices in the following way [5]:

$$
\begin{aligned}
& D|1\rangle\left\langle 1\left|=\left[1-(1-p)\left(1-e^{-A t}\right)\right]\right| 1\right\rangle\left\langle 1\left|+(1-p)\left(1-e^{-A t}\right)\right| 0\right\rangle\langle 0|, \\
& D|1\rangle\left\langle 0\left|=e^{i \omega t-G t}\right| 1\right\rangle\langle 0|, \\
& D|0\rangle\left\langle 1\left|=e^{-i \omega t-G t}\right| 0\right\rangle\langle 1|, \\
& D|0\rangle\left\langle 0\left|=p\left(1-e^{-A t}\right)\right| 1\right\rangle\left\langle 1\left|+\left[1-\left(1-e^{-A t}\right) p\right]\right| 0\right\rangle\langle 0| .
\end{aligned}
$$

The parameters $A$ and $G$ are related to energy relaxation time $\tau_{E}$ and dephasing time $\tau_{D}[21]: A=1 / \tau_{E}, G=1 / \tau_{D}$. In order for the mapping to correspond to a physically plausible completely positive transformation, $A$ and $G$ need to oblige the inequalities $G \geq A / 2 \geq 0$ [21]. The 'thermal weight' $p$ is related to the temperature $T$ as $p=\exp (-\omega / 2 T) /[\exp (-\omega / 2 T)+\exp (\omega / 2 T)]$. Notice, that the long-time limit of any qubit state evolving under the Davies map is a Gibbs state with all its physical consequences [11].

Recall that a density matrix $X$ of a state $\rho$ is called an $\mathrm{X}$-matrix (or $\mathrm{X}$-state) if it has the following form:

$$
X=\left(\begin{array}{cccccc}
a_{1} & & & & & z_{1}^{*} \\
& \ddots & & & . & \\
& & a_{n} & z_{n}^{*} & & \\
& & z_{n} & b_{n} & & \\
& . \cdot & & & \ddots & \\
z_{1} & & & & & b_{1}
\end{array}\right),
$$


i.e. the only (possibly) nonzero elements are located on its diagonal or anti-diagonal. In order for $X$ to be a correctly defined density matrix its coefficients need to satisfy $\sum_{i=1}^{n}\left(a_{i}+b_{i}\right)=1$ and $\left|z_{i}\right| \leq \sqrt{a_{i} b_{i}}$ for all $i$. It has been proven in reference [9] that genuinely multi-partite concurrence of an X-matrix is given by

$$
C_{G M}(X)=2 \max \left\{0,\left|z_{1}\right|-w_{1}, \ldots,\left|z_{n}\right|-w_{n}\right\}
$$

with $w_{j}=\sum_{k=1, k \neq j}^{n} \sqrt{a_{k} b_{k}}$. It is interesting to note that if $C_{G M}(X)$ is positive then there is only one $j$ for which $C_{G M}(X)=2\left(\left|z_{j}\right|-w_{j}\right.$ ) (i.e. only one element realizes maximum in equation (3). Indeed, suppose that for some indices $j_{1} \neq j_{2}$ we have $0 \leq\left|z_{j_{1}}\right|-w_{j_{1}}=\left|z_{j_{2}}\right|-w_{j_{2}}$. Expanding terms $w_{j 1}$ and $w_{j 2}$ and reducing summands appearing on both sides yields $0<\left|z_{j_{1}}\right|-\sqrt{a_{j_{2}} b_{j_{2}}}=\left|z_{j_{2}}\right|-\sqrt{a_{j_{1}} b_{j_{1}}}$. Using the inequality between $\left|z_{j}\right|$ and $\sqrt{a_{j} b_{j}}$ we further get $\left|z_{j_{2}}\right|-\sqrt{a_{j_{1}} b_{j_{1}}} \leq \sqrt{a_{j_{2}} b_{j_{2}}}-\left|z_{j_{1}}\right|$, which leads us to a contradiction.

Further we will investigate a time evolution of non-interacting qubits prepared initially as maximally entangled X-matrices. We assume that only one among qubits without lost of generality we assume that the first one - is weakly coupled to the environment and its evolution is described by Davies map. In our model other qubits do not interact with the environment, their evolution is free and governed by the two-level Hamiltonians $H_{Q}$. Under such assumptions the X-form of the initial state remains preserved in the evolution and its time dependent $\mathrm{X}$-form reads as

$$
X(t)=\left(\begin{array}{cccccc}
a_{1}(t) & & & & & z_{1}(t)^{*} \\
& \ddots & & & . \cdot & \\
& & a_{n}(t) & z_{n}(t)^{*} & & \\
& & z_{n}(t) & b_{n}(t) & & \\
& . \cdot & & & \ddots & \\
z_{1}(t) & & & & & b_{1}(t)
\end{array}\right),
$$

with coefficients $a_{j}(t), b_{j}(t), z_{j}(t)$ given by the following formulas:

$$
\begin{aligned}
& a_{j}(t)=a_{j}(0)(1-u(t))+\frac{u(t) p}{1-p} b_{n-j-1}(0) \\
& b_{j}(t)=a_{n-j-1}(0) u(t)+\left(1-\frac{u(t) p}{1-p}\right) b_{j}(0) \\
& z_{j}(t)=\exp (-G t) \exp \left(-i \omega_{j}\right)
\end{aligned}
$$

where $u(t)=(1-p)[1-\exp (-A t)]$. For the analysis of the entanglement dynamics, it is necessary to investigate asymptotic behaviour of those coefficients:

$$
\begin{aligned}
\lim _{t \rightarrow \infty} z_{j}(t) & =0 \\
\lim _{t \rightarrow \infty} u(t) & = \begin{cases}(1-p) & \text { if } A>0 \\
0 & \text { if } A=0\end{cases} \\
\lim _{t \rightarrow \infty} a_{j}(t) & = \begin{cases}p a_{j}(0)+p b_{n-j-1}(0) & \text { if } A>0 \\
a_{j}(0) & \text { if } A=0\end{cases} \\
\lim _{t \rightarrow \infty} b_{j}(t) & = \begin{cases}(1-p) a_{n-j-1}(0)+(1-p) b_{j}(0) & \text { if } A>0 \\
b_{j}(0) & \text { if } A=0\end{cases}
\end{aligned}
$$




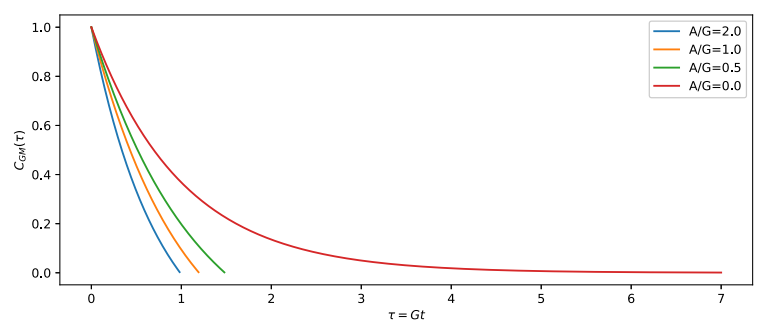

Fig. 1. Genuinely multi-partite concurrence of three-qubit GHZ state for different values of the $A / G$ ratio and $p=0.25$. Notice that for $A=0$ the entanglement sudden death never occurs.

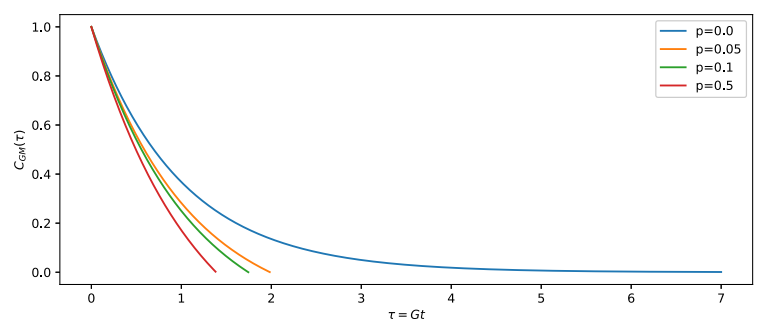

Fig. 2. Genuinely multi-partite concurrence of three-qubit GHZ state for different values of temperature $p$ and $A / G=1 / 2$. Notice that only for $p=0$ the entanglement sudden death does not occur.

An explicit form of density matrix equation (4) allows us to find an explicit and exact form of the concurrence equation (3). Note that in our model of evolution the system starting in separable state will stay separable since $\left|z_{j}(t)\right|-w_{j}(t)$ is decreasing as a function of time. That is why we will therefore focus only on system starting in the entangled state. It is not surprising that entanglement cannot be created in our model since there is no non-local interaction between qubits either direct (via qubit-qubit hamiltonian coupling) or indirect, via common heat bath mediating information exchange. In such a case, if $C_{G M}(X(0))=2\left(\left|z_{j}(0)\right|-w_{j}(0)\right)$ then $C_{G M}(X(t))=2 \max \left\{0,\left|z_{j}(t)\right|-w_{j}(t)\right\}$ for all times $t$ - otherwise we would have $0<\left|z_{j_{1}}(t)\right|-w_{j_{1}}(t)=\left|z_{j_{2}}(t)\right|-w_{j_{2}}(t)$ for some $t>0$, which would contradict our previous observation.

In the case of the dissipative environment (i.e. $A>0$ ) the system undergoes the entanglement sudden death (ESD) at $p>0$, as $\lim _{t \rightarrow \infty}\left|z_{j}(t)\right|-w_{j}(t)<0$ whereas for the case of purely dephasing environment with $A=0$, the situation can be different. If $C_{G M}(X(0))=2\left(\left|z_{j}(0)\right|-w_{j}(0)\right)$ and $w_{j}(0)=0$, the ESD does not occur and the entanglement decays asymptotically. In particular, these conclusions are applicable for the $N$-qubit GHZ states. It is presented in Figure 1 where we plot the concurrence $C_{G M}$ as a function of time for a simplest but otherwise generic case of three qubits in a GHZ-state. There is a qualitative difference between pure dephasing and the dissipative systems indicated by entanglement sudden death phenomenon. Obviously, with increasing of temperature $p$ the ESD occurs earlier as presented in Figure 2.

\section{Summary}

In our work, we attempt to simplify an otherwise highly complex problem of multipartite entanglement dynamics of open quantum systems and limit our consideration 
to (i) a very peculiar class of states - the X-states, (ii) a very peculiar type entanglement - genuine multi-partite and (iii) - last but not least - we investigate local decoherence which, however, is modelled by a very general Davies approximation. Utilizing exact result for a concurrence quantifying the entanglement we show a qualitative difference between dissipative and purely dephasing environments indicated by an existence of the (genuine multi-partite) entanglement sudden death phenomenon. We hope that our studies not only supplement our earlier studies of quantum information effects in a presence of thermal decoherence but also serve as a modest contribution to understanding multi-partite entanglement.

The work has been supported by the NCN grant 2015/19/B/ST2/02856.

Open Access This is an open access article distributed under the terms of the Creative Commons Attribution License (http://creativecommons.org/licenses/by/4.0), which permits unrestricted use, distribution, and reproduction in any medium, provided the original work is properly cited.

\section{References}

1. M. Schlosshauer, Decoherence and the quantum-to-classical transition (Springer, Berlin, Heidelberg, 2007)

2. R. Horodecki, P. Horodecki, M. Horodecki, K. Horodecki, Rev. Mod. Phys. 81, 865 (2009)

3. A. Furusawa, P. van Loock, Quantum teleportation and entanglement (Wiley, Weinheim, 2011)

4. L. Aolita, F. de Melo, L. Davidovich, Rep. Prog. Phys. 78, 042001 (2015)

5. M. Michael Walter, D. Gross, J. Eisert, Multi-partite entanglement, arXiv:1612.02437 (2017)

6. A. Acín, D. Bruß, M. Lewenstein, A. Sanpera, Phys. Rev. Lett. 87, 040401 (2001)

7. O. Gühne, G. Tóth, Phys. Rep. 474, 1 (2009)

8. M. Hofmann, T. Moroder, O. Gühne, J. Phys. A: Math. Theor. 47, 155301 (2014)

9. S.M. Hashemi Rafsanjani, M. Huber, C.J. Broadbent, J.H. Eberly, Phys. Rev. A 86, $062303(2012)$

10. E.B. Davies, Quantum Theory of Open Systems (Academic Press, London, 1976)

11. H. Spohn, J.L. Lebowitz, in Irreversible Thermodynamics for Quantum Systems Weakly Coupled to Thermal Reservoirs (John Wiley \& Sons, Inc., 2007), p. 109

12. D. Kłoda, J. Dajka, Quant. Inf. Process. 14, 135 (2015)

13. K. Lendi, A.J. van Wonderen, J. Phys. A: Math. Theor. 40, 279 (2007)

14. J. Dajka, M. Mierzejewski, J. Łuczka, R. Blattmann, P. Hänggi, J. Phys. A: Math. Theor. 45, 485306 (2012)

15. J. Dajka, J. Luczka, Phys. Rev. A 87, 022301 (2013)

16. J. Dajka, J. Łuczka, Quant. Inf. Process. 15, 2661 (2016)

17. M. Łobejko, J. Łuczka, J. Dajka, Phys. Rev. A 91, 042113 (2015)

18. J. Dajka, J. Łuczka, P. Hänggi, Quant. Inf. Process. 10, 85 (2011)

19. M Szeląg, J. Dajka, E. Zipper, J. Łuczka, Acta Phys. Pol. B 39, 1177 (2008)

20. J. Dajka, D. Kłoda, M. Łobejko, J. Sładkowski, PLoS One 10, 1 (2015)

21. W. Roga, M. Fannes, K. Zyczkowski, Rep. Math. Phys. 66, 311 (2010)

22. R. Alicki, K. Lendi, Quantum Dynamical Semigroups and Applications, Lecture Notes in Physics (Springer, Berlin, Heidelberg, 2007) 